\title{
Lévy diffusion as an effect of sporadic randomness
}

\author{
Mauro Bologna, ${ }^{1}$ Paolo Grigolini, $, 2,3$ and Juri Riccardi ${ }^{4}$ \\ ${ }^{1}$ Center for Nonlinear Science, University of North Texas, P.O. Box 5368, Denton, Texas 76203 \\ ${ }^{2}$ Istituto di Biofisica del Consiglio Nazionale delle Ricerche, Via San Lorenzo 26, 56127 Pisa, Italy \\ ${ }^{3}$ Dipartimento di Fisica dell'Università di Pisa, Piazza Torricelli 2, 56127 Pisa, Italy \\ ${ }^{4}$ ENEL SpA Struttura Ricerca-Area Generazione, Via A. Pisano 120, 56100 Pisa, Italy
}

(Received 21 June 1999)

\begin{abstract}
The Lévy diffusion processes are a form of nonordinary statistical mechanics resting, however, on the conventional Markov property. As a consequence of this, their dynamic derivation is possible provided that (i) a source of randomness is present in the corresponding microscopic dynamics and (ii) the consequent process of memory erasure is properly taken into account by the theoretical treatment. [S1063-651X(99)04812-6]

PACS number(s): 05.40.Fb, 02.50.-r, 05.60.-k
\end{abstract}

\section{INTRODUCTION}

The theoretical treatment of anomalous diffusion, namely diffusion processes either faster or slower than ordinary Brownian diffusion, is an active field of research. A well known case of superdiffusion is given by the diffusion processes of Lévy type [1]. The reader can find an exhaustive discussion of the recent literature on this subject in excellent review papers [2-5]. However, we would like to draw the reader's attention also to some key papers of the literature on this subject [6-18]. We plan to adopt a dynamic [19,20] rather than a probabilistic approach [6-18]. To make the significance of this purpose more transparent, it is convenient to compare what we mean by dynamic to the conventional probabilistic treatment, either resting on the Lévy flight or the Lévy walk method.

\section{A. Lévy flight and Lévy walk}

Both the Lévy flight and the Lévy walk method are based on a totally probabilistic treatment. The Lévy flight method is based on the assumption that at regular time intervals a space transition of arbitrarily large intensity might take place. With the Lévy walk, on the contrary, the jumps over larger distances take place in larger times. This property makes these processes non-Markovian and consequently the derivation of Lévy diffusion more delicate than from within the Lévy flight perspective. This is easily realized, for instance, by using the continuous time random-walk formalism $[6,14]$ and expressing the time evolution for the probability that the particle is at a given space location at a given time by means of the equivalent generalized master equation, see also $[15,16]$. It is then easily seen that the case where the waiting time distribution is characterized by a finite time scale yields immediately a Markov process in the long-time limit, and the anomalous diffusion properties only depend on the long-range nature of the displacement per step distribution. In Sec. II of this paper we shall study a physical condition of the same kind. Some special attention has to be devoted therefore to the Lévy walk condition, since it shares with the dynamic approach (see Sec. IC for a more precise definition of this approach) a long-time memory, which has to be properly erased to establish in the long-time limit the conditions for Lévy statistics.

\section{B. From the probabilistic to the dynamic approach}

The dynamic approach, whose precise meaning will be discussed in Sec. IC, is still somewhat obscure due to some conflicting aspects of the recent theoretical derivations used to realize this goal. This is so, in spite of the fact that the explicit adoption of techniques derived by the random walk literature yields a satisfactory derivation of the processes of Lévy diffusion from within the theoretical framework of probabilistic treatments $[7,8,4,5]$. The purpose of this paper is that of affording a unified perspective with no internal contradictions. Here we limit ourselves to pointing out some aspects of the Lévy walk method which must be retained by the dynamic approach.

First of all, as was made clear by the work of Klafter and Zumofen [17] and Zumofen and Klafter [18] (see also the report of Klafter, Zumofen, and Schlesinger [5]), we have to point out that the process of Lévy diffusion can be derived from within a dynamic perspective if the so-called Lévy walk view is adopted. This means a trajectory moving with constant velocity along a straight line for an extended time and from time to time making abrupt direction changes. The time of sojourn in one of these straight paths is characterized by the probability density function

$$
\psi(t)=\frac{(\mu-1) T^{\mu-1}}{(T+t)^{\mu}},
$$

where $T /(\mu-2)$ denotes the mean waiting time. The renormalization-group method, as illustrated by Zaslavsky [21], affords a reliable way of fixing the time asymptotic form of Eq. (1) and, notably, the power index $\mu$ in terms of the rescaling properties of the fractal region at the border between chaotic sea and stability islands. The theorem of $\mathrm{Kac}$ [22] ensures that the first moment of $\psi(t)$ is finite. This important theorem refers to the distribution of the Poincare recurrence times under the crucial condition that the system under study is ergodic. Zaslavsky [21] noticed that when a stability island is imbedded within the chaotic sea, the distribution of Poincaré recurrence times becomes equivalent to the distribution of the times of sojourn at the border between chaotic sea and stability island. This is so because a trajectory moving from a given small portion of the chaotic sea 
through a fast diffusion process arrives at the fractal region and it sticks to it for an extended time before returning to the departure region. Thus, in full accordance with the Kac theorem, the renormalization-group theory yields $\mu>2$, thereby insuring that the first moment of this distribution is finite, and consequently that also the distribution of the Poincaré recurrence times is finite.

We note that all this supports the asymptotic form of Eq. (1) leaving, though, the impression that its short-time structure is arbitrary. It is not so. As proved in a recent work [23], the whole structure of Eq. (1) is dictated by the principle of entropy maximization provided that the entropy used is that of Tsallis [24] rather than the conventional Gibbs-Shannon entropy. Note that the explicit form of Eq. (1) that we are using is fixed, of course, by both the normalization condition and the condition that the first moment is finite and that its value is $T /(\mu-2)$. In conclusion, the form of Eq. (1) is a unique analytical expression determined by the joint use of dynamics, renormalization-group technique, and entropy.

We hope that with no sacrifice of the most important ingredients behind the dynamic derivation of the Lévy diffusion process, we can restrict our investigation to the onedimensional case. In this condition the role of deterministic and dynamical generator of the Lévy diffusion can be properly played by the intermittent map [25] used by Zumofen and Klafter [18] and by Klafter and Zumofen [17]. This is a map with the same algorithmic complexity as the Manneville map [26], the complexity of which has been studied by Gaspard and Wang [27] by means of the Kolmogorov-Sinai entropy.

In the Hamiltonian model of Zavslasky, the derivation of the diffusion processes of Lévy rests on the microcanonical conditions. This means that the kinetic energy of the flight process is fixed. Consequently, the one-dimensional version of this Hamiltonian perspective yields the important property that only two velocity states exist, one with velocity $W$ and one with velocity $-W$. As a consequence of the onedimensional assumption, therefore, we are allowed to use the key relation [25]

$$
\Phi_{\xi}(t)=\frac{\mu-2}{T} \int_{t}^{\infty}\left(t^{\prime}-t\right) \psi\left(t^{\prime}\right) d t^{\prime},
$$

which is equivalent to

$$
\psi(t)=\frac{T}{\mu-2} \frac{d^{2}}{d t^{2}} \Phi_{\xi}(t) .
$$

Equations (2) and (3) relate to one another the physical properties $\psi(t)$ and $\Phi_{\xi}(t)$. The former property, $\psi(t)$, is the probability density function of sojourn times, which, as earlier stressed, has an inverse power-law form [see Eq. (1)]; the latter, $\Phi_{\xi}(t)$, is the stationary correlation function of the dichotomous variable $\xi$, playing the role of a velocity with only two possible values, $W$ and $-W$. The function $\Phi_{\xi}(t)$ is determined by the statistical properties of the velocity of the paths moving with constant velocity and without changing direction. We note that Eq. (3) establishes that $\psi(t)$ is proportional to the second-order time derivative of the function $\Phi_{\xi}(t)$, thereby implying, as a consequence of Eq. (1), that for $t \rightarrow \infty$ the decay of $\Phi_{\xi}(t)$ is proportional to $1 / t^{\beta}$ with $\beta$ $=\mu-2$. The region of interest for us is that in which the first moment of $\psi(t)$ is finite (so as to fit the Kac theorem) and the second moment is divergent so as to prevent the system from falling in the attraction basin of the central limit theorem. Consequently, we restrict our analysis to the interval $0<\beta<1$. Note that Eq. (2) is exact (see [25]) if the assumption is made that the time interval between the transition from one to the other velocity state is instantaneous. To help the reader to understand the main conclusion of this paper, we have also to make another preliminary remark. The correlation function $\Phi_{\xi}(t)$ is a stationary property [19], implying the existence of an invariant, or equilibrium, distribution. A genuinely dynamic approach to the Lévy processes, consistent with the ergodic assumption behind the Kac theorem, implies that this equilibrium distribution is established by a single trajectory, provided that this trajectory runs for an unlimited amount of time. The lack of a finite microscopic time scale makes this condition difficult to realize in practice, and it is probably one of the sources of the conflicting views that will be discussed in this paper.

\section{The dynamic approach}

The general program of the dynamic approach to statistical mechanics is illustrated in a series of recent papers [2831]. We are very close to the program of Ref. [31]. The ambitious purpose of these authors is to derive an important equation such as the Fokker-Planck equation without using any statistical assumption whatsoever, so as to reverse the ordinary path from thermodynamics to statistical mechanics. In other words, the path to follow moves from dynamics and reaches the level of statistical mechanics using only deterministic randomness with no recourse to thermodynamics, this being the last step, stemming from the dynamically generated statistical equilibrium distributions.

The authors of [20] adopted the same perspective to move from dynamics to Lévy statistics. The authors of Ref. [20] found that the density distribution $\sigma(x, t)$ of the variable $x$ driven by a process described by Eq. (2) obeys the equation of motion [20]

$$
\frac{\partial}{\partial t} \sigma(x, t)=\left\langle\xi^{2}\right\rangle \int_{0}^{t} \Phi_{\xi}\left(t^{\prime}\right) \frac{\partial^{2}}{\partial x^{2}} \sigma\left(x, t-t^{\prime}\right) d t^{\prime} .
$$

Within the context of a dynamic approach to the Lévy processes, this equation should be given special attention, since no explicit use of probabilistic arguments was made to derive it [20]. However, no general solution of it is available, and the emergence of the Lévy diffusion out of it rests on an approximation which has been questioned [32,33]: Different approximations to the solution of Eq. (4) lead to different statistical processes. The interested reader is referred to the work of Ref. [20] for the derivation of Eq. (4). Here we limit ourselves to noticing that this equation is exact under the condition that the velocity variable is dichotomous and the initial distribution is a Dirac $\delta$ centered at $x=0$. Thus, there is an intimate relation between Eq. (2) and Eq. (4). 


\section{Purpose and outline of the paper}

The main purpose of this paper is that of stressing that the intimate connection between Eq. (2) and Eq. (4) does not leave room for a solution implying a memory infinitely extended in time. In other words, we want to prove that the adoption of a Markovian perspective, although apparently incompatible with the time convolution of Eq. (4), is dictated by the steady action of the randomness corresponding to the transition from one state to the other of variable velocity. Consequently, the Markov structure emerging from Eq. (4) according to the prescriptions of [20] cannot be misconstrued as an undue approximation. This is rather an ingenuous way of establishing a physical condition fitting the result of an earlier, and crucial, research work. Gaspard and Wang [27] prove that in the long-time limit, the Manneville map becomes equivalent to the Bernouilli shift map. This is a consequence of the fact that the repeated exit and reentering into the laminar region results in a memory erasure. As we shall see, this is the main reason why the final state is as Markov as a genuine Lévy process must be.

In other words, we plan to make randomness emerge from the dynamic approach, resting on Eq. (4), so as to render this dynamic approach equivalent to the Lévy walk perspective. The purpose of this paper is to show that the Markov property necessary to derive the process of Lévy diffusion is not arbitrary, but rather corresponds intimately to the nature of the dynamic process resulting in Eq. (4). This is so because Eq. (4) implies the dichotomous nature of the variable $\xi$. The condition where for $t \rightarrow \infty$ the decay of $\Phi_{\xi}(t)$ is proportional to $1 / t^{\beta}$ with $\beta=\mu-2$ means that the process is deterministic for an overwhelming amount of time. There exists an intimate equivalence between Eq. (2), in this physical condition, and intermittent maps. Randomness shows up only when the trajectory reaches the border between the laminar and chaotic region [26]. At this crucial stage there are only two possible events, either a jump from the original into the other laminar region, corresponding to a distinct velocity state, or the jump back to the original laminar region, namely, the original velocity state [18]. At this stage dynamics are essentially indistinguishable from the time evolution of the Bernouilli shift map, whose connection with thermodynamics and statistical mechanics has been recently clearly illustrated by Zaslavsky [21]. This means that randomness is a rare event and it is in fact the reason why we have adopted the concept of sporadic randomness. The main purpose of this paper is that of making a choice between two distinct ways of solving Eq. (4), based on the criterion that the right solution must reflect this sporadic randomness.

The outline of this paper is as follows. In Sec. II we review the arguments used in an earlier paper [34], to derive a process of Lévy diffusion by means of a generalized master equation. In Sec. III, using the calculation illustrated in the Appendix, we show that the same result is derived from a master equation which looks like the Markov approximation of that of Sec. II. In Sec. IV we review, in the light of the perspective established in this paper, the method used in Ref. [20] to assign to Eq. (4) a Markov structure. Some final conclusions are made in Sec. V.

\section{THE GENERALIZED MASTER EQUATION}

The first step of our approach rests on the use of the generalized master equation of Ref. [35]. This equation reads

$$
\frac{\partial}{\partial t} \sigma(x, t)=\int_{0}^{t} d t^{\prime} \int_{-\infty}^{\infty} \kappa\left(x-x^{\prime}, t-t^{\prime}\right) \sigma\left(x^{\prime}, t^{\prime}\right) d x^{\prime}
$$

where

$$
\kappa(x, t) \equiv \pi(x, t)-\delta(x) \int_{-\infty}^{\infty} d x^{\prime} \pi\left(x^{\prime}, t\right)
$$

and $\pi(x, t)$ denotes the probability for the particle to make a jump by a distance $x$ at time $t$. This equation is very general and is expected to be compatible with the description of highly non-Markovian processes such as that corresponding to Eq. (4) with the nonintegrable correlation function generated by Eqs. (1) and (2). The intimate connection between these two equations will be discussed in Sec. IV. Here we limit ourselves to stressing that the asymptotic regime of $\sigma(x, t)$, as given by Eq. (5), can be studied without making explicitly the Markov approximation. In fact, using the property that this equation is convoluted in both space and time variables, we get for the Fourier-Laplace transform of $\sigma(x, t)$, denoted by $\hat{\sigma}(k, s)$, the following expression:

$$
\hat{\sigma}(k, s)=\frac{1}{s-\hat{\kappa}(k, s)},
$$

where, of course, $\hat{\kappa}(k, s)$ denotes the Fourier-Laplace transform of $\kappa(x, t)$. As pointed out in Sec. I, the dynamic approach to Lévy statistics that we are considering is related to the Lévy walk condition. This means that a transition of length $|x|$ implies a time $t=|x| / W$. In conclusion, we are forced to make the following choice for $\pi(x, t)$, with $t>0$ :

$$
\pi(x, t)=\psi(t) \delta(|x|-W t)
$$

The authors of Ref. [34] studied the asymptotic regime of Eq. (7), supplemented by Eq. (8), searching for the rescaling condition

$$
s \propto k^{\alpha},
$$

with $\alpha>1$. This is a reasonable assumption, since in the asymptotic limit the second moment is known [34] to yield

$$
x \approx t^{2 H}
$$

with

$$
H=1-\beta / 2 \text {. }
$$

On the other hand, the rescaling of Eq. (9) suggests that the condition $\alpha=1 / H$ might apply, thereby resulting in the property $\alpha>1$, which is essential for the calculations aiming at establishing the exact dependence of $\alpha$ on $\beta$.

With straightforward calculations it is shown [34] that in the asymptotic limit Eq. (7) and Eq. (8) yield

$$
\alpha=\beta+1
$$

and

$$
\hat{\sigma}(k, s)=\frac{1}{s+b|k|^{\alpha}},
$$


with

$$
b \equiv \Gamma(1-\alpha) \frac{(W T)^{\alpha}}{T}(\alpha-1) \cos \left(\frac{\alpha \pi}{2}\right) .
$$

Note that this is the Laplace transform of the following equation of motion:

$$
\frac{\partial}{\partial t} \hat{\sigma}(k, t)=-b|k|^{\alpha} \hat{\sigma}(k, t)
$$

This means that the asymptotic regime of the generalized master equation of Eq. (5) is a process of diffusion with a genuinely Lévy nature [2].

We note that Eq. (12) means the rescaling,

$$
H=\frac{1}{1+\beta},
$$

which is different from that of Eq. (11). The difference between the two rescalings is a fact of crucial importance deserving proper comments. We note that the rescaling of Eq. (11) is somewhat ambiguous since it refers to the dynamics of Eq. (7). As pointed out in Ref. [20], as well as in the earlier work of Zumofen and Klafter [18] and Klafter and Zumofen [17], the diffusion process described by Eq. (7) consists of a central part and a propagation front signaled by two sharp peaks. At time $t$, a particle leaving the origin $x$ $=0$ at $t=0$ cannot be found at a distance from the origin larger than $W t$. This has the effect of producing an accumulation of particles at the front of the diffusion process, namely at $x= \pm W t$. This is the origin of the two ballistic peaks of the propagation front. At earlier times the initial distribution, concentrated at $x=0$, splits into these two ballistic peaks and the region between the two peaks is empty. Due to the effect of sporadic randomness, some trajectories leave the propagation front and the population of the central part steadily increases in time, while the peak intensity, proportional to the correlation function $\Phi_{\xi}$, slowly decreases. Note that this means that the diffusion process cannot be described by a single rescaling. The peaks of the propagation front rescale with $H=1$, a fact implying a diffusion faster than that predicted by the rescaling of Eq. (11). The rescaling of the central part is properly expressed by Eq. (16). The calculations leading to Eq. (15) refer to a physical condition where the intensity of the ballistic peaks is negligible, so that the rescaling of Eq. (16) only reflects the diffusion properties of the distribution central part. On the contrary, the rescaling of Eq. (11) is a sort of balance between the fast rescaling of the propagation front and the rescaling of the central part of the distribution $\sigma(t)$, which is in fact slower than the rescaling of Eq. (16). In conclusion, the discrepancy between the rescaling of Eq. (11) and the rescaling of Eq. (16) reflects the fact that the derivation of the Lévy process from within the Lévy walk perspective rests on a deep conflict between the dynamic properties still present within the Lévy walk perspective and a merely probabilistic treatment.

We want to make a further remark, concerning the derivation of a Lévy process from the generalized master equation of Eq. (5). We note that the Lévy processes are a form of Markov statistics [1] thereby implying that the asymptotic regime of the dynamic process described by Eq. (5) involves a process of memory erasure. This paper is devoted to proving that Eq. (4), in spite of its non-Markovian structure, yields an asymptotic regime whose statistics are determined by this process of memory erasure.

\section{THE MARKOV MASTER EQUATION}

As pointed out at the end of Sec. II, the processes of Lévy diffusion are Markovian. Therefore, it is convenient to discuss their derivation from a Markov master equation. This discussion will serve the useful purpose of proving that the master equation is, in a sense, a bridge between the dynamic treatment and the diffusion regime. The latter is the subject of thermodynamical arguments and the former rests, in the theoretical picture adopted in this paper, on classical mechanics. Thus, the master equation can also be regarded as an important bridge between mechanics and thermodynamics. In the continuous representation the master equation reads (see [2], [36], and [34])

$$
\frac{\partial}{\partial t} \sigma(x, t)=\int_{-\infty}^{\infty} K\left(x-x^{\prime}\right) \sigma\left(x^{\prime}, t\right) d x^{\prime},
$$

where

$$
K(x)=\Pi(x)-\delta(x) \int_{-\infty}^{\infty} \Pi\left(x^{\prime}\right) d x^{\prime}
$$

As far as the transition probability $\Pi(x)$ is concerned, we adopt the result of the entropic analysis of Ref. [23]. Thus we write

$$
\Pi(x)=\frac{1}{T} \frac{1}{W} \psi\left(\frac{|x|}{W}\right)=\frac{(\mu-1) T^{\mu-1} W^{\mu-1}}{(T W+|x|)^{\mu}},
$$

with $2<\mu<3$. In fact, the theoretical work of Ref. [23] proves that the maximization of the Tsallis entropy [24] with entropic index $q=1+1 / \mu$ yields for $\Pi$ an inverse power-law form with index $\mu$, provided that a transition of length $|x|$ is supposed to be related to the time $t$ by $|x|=W t$. The adoption of this entropic argument changes Eq. (17) into

$$
\begin{aligned}
\frac{\partial}{\partial t} \sigma(x, t)= & \frac{(\mu-1)(T W)^{\mu-1}}{T}\left[\int_{-\infty}^{\infty} \frac{\sigma\left(x^{\prime}, t\right) d x^{\prime}}{\left(T W+\left|x-x^{\prime}\right|\right)^{2+\beta}}\right. \\
& \left.-\int_{-\infty}^{\infty} \frac{d x^{\prime}}{\left(T W+\left|x^{\prime}\right|\right)^{2+\beta}} \sigma(x, t)\right],
\end{aligned}
$$

where $\mu=2+\beta$ with $0<\beta<1$. As shown in the Appendix, the Fourier transform analysis of Eq. (20) proves that in the asymptotic regime $(k W T \ll 1)$ this equation is equivalent to the process of Lévy diffusion of Eq. (15) where the parameter $b$ reads

$$
b=2 \cos \left(\frac{\pi \alpha}{2}\right) \Gamma(1-\alpha) \frac{(W T)^{\alpha}}{T} .
$$


In other words, we derive a result equivalent to that of Sec. II. Note that in the limiting case $T=0$ the term within the square brackets of Eq. (20) becomes the regularized [37] form of

$$
\int_{-\infty}^{+\infty} \frac{1}{\left|x-x^{\prime}\right|^{2+\beta}} \sigma\left(x^{\prime}, t\right) d x^{\prime}
$$

and, in this sense, coincides with the expression found by Seshadri and West [9]. Note that keeping $T>0$ makes it possible for Eq. (20) to cross the critical condition $\beta=1$ without meeting the divergence corresponding to the Lévy prescription, namely the divergence of $b$ of Eq. (14) at $\alpha$ $=2$. The result of Eq. (20) can be used also to study the region $\beta>1$ corresponding to the attraction basin of the ordinary central limit theorem.

It is important to observe that the Markovian master equation under study here can be derived from the generalized master equation of Eq. (5) by using the Markov condition:

$$
\Pi(x)=\int_{0}^{\infty} \pi\left(x, t^{\prime}\right) d t^{\prime} .
$$

This is an aspect of crucial importance. In fact, Eq. (23) yields Eq. (19), showing that the Markov property makes it possible to establish an important connection between dynamics and thermodynamics. The Markov perspective adopted in this section is essential to establishing the key connection between the structure of Eq. (19), resulting from the adoption of entropic arguments, and the structure of Eq. (1), generated by the adoption of the renormalization-group arguments, which, in turn, reflect genuinely Hamiltonian properties [21]. Note that we refer to Eq. (23) as a Markov condition rather than as a Markov approximation. This is so because the term "approximation" suggests a given departure from the exact solution or, in other words, an error whose intensity must be defined. We see, on the contrary, that the asymptotic regime of Eq. (20) coincides with that of Eq. (5) if the latter equation is supplemented by the crucial condition of Eq. (8), mirroring the dynamics illustrated in the Introduction.

\section{THE EXACT DIFFUSION EQUATION AND THE MARKOV REGIME}

Is there a connection between Eq. (4) and Eq. (20)? It is evident that this connection would be established by the exact solution of Eq. (4) if this yielded, in the asymptotic time limit, a diffusion process of the Lévy kind. It has to be pointed out, however, that finding an exact solution of Eq. (4) is not easy. In the literature we find only solutions of Eq. (4) based on approximations [20,32,33]. In Ref. [20] a solution of Eq. (4) was found, with a Markov character, and corresponding to Eq. (20) with only the first of the two terms between the square brackets on the right-hand side of this equation. In Ref. [32] a non-Markov solution has been discussed, which has been later judged to be the correct solution [33].

The purpose of this section is that of discussing these two proposed solutions in the light of the sporadic randomness illustrated in Sec. I. In the work of [32], it was argued that an analytical solution of Eq. (4) can be found, based on the fact that a fractional derivative in time emerges from the righthand side of Eq. (4) if the correlation function $\Phi_{\xi}(t)$, which should fulfill the normalization condition $\Phi_{\xi}(0)=1$, is replaced by a function like const $/ t^{\beta}$. This means a function with the same long-time property as the original correlation function, breaking, however, the normalization condition at $t=0$. This approximation results in very appealing mathematical properties. In fact, it has the nice effect of resulting in a process with infinite memory and in an analytical expression for the effects that this infinitely extended memory has on diffusion. The adoption of this approximation yields [33] the rescaling of Eq. (11). It is straightforward to prove that this rescaling can be obtained from the Fourier-Laplace transform of Eq. (4),

$$
\hat{\sigma}(k, s)=\frac{\left\langle\xi^{2}\right\rangle}{s+\hat{\Phi}_{\xi}(s) k^{2}},
$$

where $\hat{\Phi}_{\xi}(s)$ is the Laplace transform of the correlation function $\Phi_{\xi}(t)$. To derive the rescaling of Eq. (11) we have to replace in Eq. (24) $\hat{\Phi}_{\xi}(s)$ with $s^{\beta-1}$ (see [38]). This rescaling, however, conflicts with the numerical observation of Ref. [20], which results in a different rescaling, corresponding to that of Eq. (16). The discussion of Sec. II sheds light on the origin of this rescaling, different from that of Eq. (11). It seems to be evident to us that the study of the asymptotic properties of Eq. (24) resting on the limiting condition $\lim _{s \rightarrow 0} \hat{\Phi}_{\xi}(s)=$ const $\times s^{\beta-1}$ loses any dependence on the key parameter $T$, and with it, on the fact that there exists a propagation front moving with finite velocity. This explains why the same method of time asymptotic analysis applied to Eq. (7) yields the correct rescaling. This is so because in this case the Lévy walk nature of the process under study is retained by the kernel $\kappa(x, t)$ due to the wise choice made for $\pi(x, t)$ in Eq. (8).

In conclusion, we are convinced that the solution implying the existence of an infinitely extended memory conflicts with the numerical treatment of the diffusion process resulting from the fluctuations of the dichotomous variable $\xi$ with a nonintegrable correlation function $\Phi_{\xi}(t)$. This is so because the steady action of sporadic randomness has the effect of producing a Markov statistics, although this occurs in the long-time limit. How can we make the rescaling of the central part of the distribution $\sigma(t)$ become compatible with the effect of sporadic randomness and with the predictions of the Lévy-Gnedenko theorem [39] in the long-time limit? The most direct way to realize the correct rescaling of the central part of the distribution and to make the Markov property emerge is that of assuming that

$$
\sigma\left(x, t-t^{\prime}\right)=\frac{1}{2} \int_{-\infty}^{\infty} \delta\left(W t^{\prime}-\left|x-x^{\prime}\right|\right) \sigma\left(x^{\prime}, t\right) d x^{\prime}
$$

As pointed out earlier, if we apply this condition to the term on the right-hand side of Eq. (4), we obtain an equation of motion identical to one that would result from Eq. (20) canceling the second of the two terms within the square brackets of this equation. This interesting result implies some algebra 
based on the method of integration by parts and the properties of the delta of Dirac. More details on this calculation can be found in Ref. [20].

It is important to point out the physical meaning of the constraint of Eq. (25). This means that we imagine a condition still unaffected by randomness, since this constraint would be rigorously valid only in the case of merely ballistic motion. Yet the effect of replacing Eq. (25) into the righthand side of Eq. (4) is that of producing a Markov structure as an effect of carrying out the integration on $t^{\prime}$ in the timeconvoluted form of Eq. (4). We are convinced that the emergence of this Markovian structure is not an artifact of the approximation of Eq. (25). The error associated to this approximation is not the emergence of the Markov structure. This error is totally different in nature, and can be easily evaluated. In fact, as repeatedly pointed out earlier, this approximation has the effect of resulting only in the first term on the right-hand side of Eq. (20). The error associated to this approximation is signaled by the breaking of the norm conservation. It is evident, in fact, that the condition

$$
\int_{-\infty}^{\infty} \sigma(x, t) d x=1
$$

is fulfilled by both Eqs. (4) and (20) and that in the latter case this is a consequence of the wise structure of the master equation of Eq. (17). This is the reason why we look at the master equation of Eq. (20) as a natural bridge to cross when moving from the dynamical perspective of Eq. (4) to the final regime of the Lévy kind. As noticed in Sec. III, the emergence of this final condition from Eq. (20) is made by using the mathematical approach of the Appendix, which proves that the Fourier transform of Eq. (20) yields the genuine Lévy process of diffusion of Eq. (15). We note that in the earlier work $[20,40]$ no proper attention was devoted to the crucial fact that the second term within the square bracket of Eq. (20) is essential for a proper derivation of the Lévy processes, or of the equivalent fractional derivative.

\section{CONCLUDING REMARKS}

In conclusion, we have provided a convincing demonstration of how to derive Lévy processes from within a dynamic approach. As pointed out in an earlier work [40], the Lévy process corresponds to a form of fractional calculus which can be regarded as a form of macroscopic manifestation of microscopic randomness. However, it seems to us that fractional derivatives in time have a different meaning from fractional derivatives in space. The choice made by the authors of Ref. [33] has the effect of relating the solution of Eq. (4) to a form of fractional derivative in time. It seems to us that this choice corresponds to a case where the decay of the correlation function is not originated by the sporadic action of randomness on a single trajectory. In this latter case, as shown in this paper, to obtain the correct result we are forced to make the Markov property emerge, and this is realized by adopting the trick of Eq. (25). The assumption made by the authors of Ref. [33], on the contrary, seems to imply that the decay of the correlation function is originated by a statistical distribution over a range of initial conditions. Whether or not this is compatible with the dichotomous nature of the diffu- sion generator is not quite clear to us.

Should the proof be given that the relaxation of the correlation function $\Phi_{\xi}$ can also be determined by statistics as well as by dynamics, with no conflict with the dichotomous property behind Eq. (4), we would reach the impressive conclusion that this exact equation admits two distinct classes of solution, determined by the extra information about the physical origin of the relaxation process. We are inclined to believe that the relaxation of a dichotomous variable can only be compatible with the action of a sporadic randomness. Thus the emergence of the Markov property at extremely long time is a fair reflection of the dynamical processes generating the Lévy diffusion processes, not to mention the fact that the corresponding rescaling of the central part of the distribution fits the results of the numerical calculations [20].

It is also important to stress that the repeated action of randomness is subtly related to the possibility of establishing a connection between mechanics and thermodynamics also in the case, studied in this paper, of apparently infinite memory. The random seed is given by the fact that the duration of the times of sojourn in the laminar region cannot be predicted. This is so because of the random injection into the laminar region from the chaotic part of the map [25]. This is the reason why we have to introduce probabilistic arguments within the dynamical picture of the process under study. This is also the reason why, as shown by [23], the shape of the density distribution of Eq. (1) can be predicted by using entropic arguments, provided that the nonextensive form of entropy advocated by Tsallis [24] is used. In other words, both the adoption of entropic arguments and the birth of Lévy statistics rest on the emergence of probabilistic aspects generated by a sporadic form of randomness.

Finally, we are left with the intriguing issue of the dependence of all these properties on the space dimensions. The treatment of this paper has been confined to the onedimensional case, where the crucial action of the stability islands pointed out by the theoretical analysis of Zaslavski [21] is correctly mirrored by the dichotomous nature of the fluctuating variable $\xi$. We are convinced that the essence of the present treatment can be extended to the multidimensional case, including the more realistic three-dimensional case. However, we have to recognize that this extension is not a trivial matter and that further research work has to be done to settle the technical problems triggered by the twoand three-dimensional case.

\section{APPENDIX}

We define the function

$$
D_{x}^{\alpha} e^{x}=\sum_{n=0}^{\infty} \frac{x^{n-\alpha}}{\Gamma(n+1-\alpha)} \equiv E_{\alpha}^{x} .
$$

This function is a generalization of the exponential function. In turn, this generalized exponential is used to derive the following form of the generalized trigonometric function:

$$
\sin _{\alpha} x=\frac{E_{\alpha}^{\imath x}-E_{\alpha}^{-\imath x}}{2 \imath}
$$




$$
\cos _{\alpha} x=\frac{E_{\alpha}^{\imath x}+E_{\alpha}^{-\imath x}}{2}
$$

The corresponding expansions in a power series are

$$
\sin _{\alpha} x=\sum_{n=0}^{\infty} \frac{x^{n-\alpha} \sin [(n-\alpha) \pi / 2]}{\Gamma(n+1-\alpha)}
$$

and

$$
\cos _{\alpha} x=\sum_{n=0}^{\infty} \frac{x^{n-\alpha} \cos [(n-\alpha) \pi / 2]}{\Gamma(n+1-\alpha)} .
$$

Note that the functions defined in Eq. (A1) fulfill the important relation

$$
\int x^{\alpha} e^{x} d x=\Gamma(1+\alpha) e^{-x} E_{-1-\alpha}^{x}
$$

We are now ready to address the problem raised by Eq. (20), namely, the evaluation of the Fourier transform of the function $1 /\left(T W+\left|x-x^{\prime}\right|\right)^{2+\beta}$. For this purpose we can use Eq. (A6) and we find

$$
\int_{-\infty}^{+\infty} \frac{e^{\imath k x}}{(a+|x|)^{2+\beta}} d x=2 \operatorname{Re} \int_{0}^{+\infty} \frac{e^{i k x} d x}{(a+|x|)^{1+\alpha}}=f(k) .
$$

On the other hand,

$$
\begin{aligned}
f(k)= & 2 \int_{0}^{+\infty} \frac{\cos k x}{(a+x)^{1+\alpha}} d x \\
= & 2 \Gamma(-\alpha)|k|^{\alpha} \\
& \times\left[\sin \left(\frac{\pi}{2}(1+\alpha)+|k a|\right)-\widetilde{\sin }_{\alpha}\left(\frac{\pi}{2}(1+\alpha)+|k a|\right)\right],
\end{aligned}
$$

where $a \equiv W T$ and

$$
\begin{aligned}
\widetilde{\sin }_{\alpha}\left(\frac{\pi}{2}(1+\alpha)+|k a|\right)= & \cos \left(\frac{\pi}{2}(\alpha+1)\right) \sin _{\alpha}|k a| \\
& +\sin \left(\frac{\pi}{2}(\alpha+1)\right) \cos _{\alpha}|k a| .
\end{aligned}
$$

We are interested in the case $k a \rightarrow 0$. Thus we use Eqs. (A4), (A5), and (A9) to evaluate the Fourier transform of interest keeping only the leading vanishing and diverging terms. We thus obtain

$$
\begin{aligned}
\int_{-\infty}^{+\infty} d x \frac{\exp (i k x)}{(a+|x|)^{(1+\alpha)}} \\
\approx 2|k|^{\alpha} \Gamma(-\alpha)\left[\cos \left(\frac{\pi \alpha}{2}\right)-\sin \left(\frac{\pi \alpha}{2}\right)|k a|\right. \\
\left.\quad-\frac{|k a|^{-\alpha}}{\Gamma(1-\alpha)}+\frac{|k a|^{2-\alpha}}{\Gamma(3-\alpha)}\right]
\end{aligned}
$$

In conclusion, the Fourier transform of Eq. (20) yields

$$
\frac{\partial}{\partial t} \hat{\sigma}(k, t)=c[f(k)-f(0)] \hat{\sigma}(k, t),
$$

where $f(k)$ is given by Eq. (A8) and

$$
c \equiv \frac{1}{T}(\mu-1) T^{(\mu-1)} W^{(\mu-1)}=\frac{1}{T}(\mu-1) a^{(\mu-1)},
$$

where $\mu-1=\alpha$. After some algebra we find that $f(0)$ $=2 /\left(\alpha a^{\alpha}\right)$ and the final coefficient in front of $\sigma(k, t)$ is

$2 \cos \left(\frac{\pi \alpha}{2}\right) \Gamma(-\alpha) \frac{\alpha|k a|^{\alpha}}{T}=-2 \cos \left(\frac{\pi \alpha}{2}\right) \Gamma(1-\alpha) \frac{a^{\alpha}}{T}|k|^{\alpha}$.

As a final result we get Eq. (15).
[1] P. Lévy, Théory de l'Addition des Variables Aléatoires (Gauthier-Villars, Paris, 1937).

[2] E.W. Montroll and B.J. West, in Fluctation Phenomena, 2nd ed., edited by E.W. Montroll and J.L Lebowitz, Studies in Statistical Mechanics Vol. 7 (North Holland, Amsterdam, 1987).

[3] J.-P. Bouchaud and A. Georges, Phys. Rep. 195, 12 (1990).

[4] M.F. Shlesinger, G.M. Zaslavsky, and J. Klafter, Nature (London) 363, 31 (1993).

[5] J. Klafter, M.F. Shlesinger, and G. Zumofen, Phys. Today 49 (2), 33 (1996).

[6] J. Klafter, A. Blumen, and M.F. Shlesinger, Phys. Rev. A 35, 3081 (1987).

[7] M.F. Shlesinger, B.J. West, and J. Klafter, Phys. Rev. Lett. 58, 1100 (1987).

[8] A. Blumen, G. Zumofen, and J. Klafter, Phys. Rev. A 40, 3964 (1989).
[9] V. Seshadri and B.J. West, Proc. Natl. Acad. Sci. USA 79, 4501 (1982).

[10] B. J. West and V. Seshadri, Physica A 113, 203 (1982).

[11] F. Peseckis, Phys. Rev. A 36, 892 (1987).

[12] H. C. Fogedby, Phys. Rev. Lett. 73, 2517 (1994); Phys. Rev. E 50, 1657 (1994).

[13] J. Honkonen, Phys. Rev. E 53, 327 (1996).

[14] A. Compte, Phys. Rev. E 53, 4191 (1996).

[15] R. Metzler, E. Barkai, and J. Klafter, Europhys. Lett. 46, 431 (1999).

[16] R. Metzler, E. Barkai, and J. Klafter, Phys. Rev. Lett. 82, 3563 (1999).

[17] J. Klafter and G. Zumofen, Phys. Rev. E 49, 4873 (1994).

[18] G. Zumofen and J. Klafter, Phys. Rev. E 47, 851 (1993).

[19] R. Mannella, P. Grigolini, and B.J. West, Fractals 2, 81 (1994).

[20] P. Allegrini, P. Grigolini, and B.J. West, Phys. Rev. E 54, 4760 (1996). 
[21] G.M. Zaslavsky, Physics of Chaos in Hamiltonian System (Imperial College Press, London, 1998).

[22] M. Kac, Probability and Related Topics in Physical Sciences (Interscience, New York, 1958).

[23] M. Buiatti, P. Grigolini, and A. Montagnini, Phys. Rev. Lett. 82, 3383 (1999).

[24] C. Tsallis, J. Stat. Phys. 52, 479 (1988).

[25] T. Geisel and S. Thomae, Phys. Rev. Lett. 52, 1936 (1984).

[26] P. Manneville, J. Phys. (Paris) 41, 1235 (1981).

[27] P. Gaspard and X.-J. Wang, Proc. Natl. Acad. Sci. USA 85, 4591 (1988).

[28] B.L. Holian, W.G. Hoover, and H.A. Posch, Phys. Rev. Lett. 59, 10 (1987).

[29] T. Petrosky and I. Prigogine, Chaos, Solitons and Fractals 7, 441 (1996).

[30] C. Jarzynski, Phys. Rev. Lett. 74, 2937 (1995).

[31] M. Bianucci, R. Mannella, B.J. West, and P. Grigolini, Phys. Rev. E 51, 3002 (1995).

[32] B.J. West, P. Grigolini, R. Metzler, and T.F. Nonnenmacher,
Phys. Rev. E 55, 99 (1997).

[33] R. Metzler and T.F. Nonnenmacher, Phys. Rev. E 57, 6409 (1998).

[34] G. Trefan, E. Floriani, B.J. West, and P. Grigolini, Phys. Rev. E 50, 2564 (1994).

[35] V.M. Kenkre, E.W. Montroll, and N.F. Shlesinger, J. Stat. Phys. 9, 3964 (1989).

[36] D. Bedeaux, K. Lakatos, and K. Shuler, J. Math. Phys. 12, 2116 (1971).

[37] I.M. Gelfand and G.E. Saletan, Generalized Functions:Properties and Operations (Academic Press, New York, 1964), Vol. I.

[38] I.S. Gradshteyn and I.M. Ryzhik, Table of Integrals, Series, and Products (Academic, New York, 1994).

[39] B.V. Gnedenko and A.N. Kolmogorov, Limit Distributions for Sums of Independent Random Variables (Addison-Wesley, Reading, MA, 1954).

[40] P. Grigolini, A. Rocco, and B.J. West, Phys. Rev. E 59, 2603 (1999). 